\title{
Validation and Additive Predictive Value of the Academic Research Consortium-High Bleeding Risk Criteria in Older Adults
}

\author{
Claudio Montalto ${ }^{1,2}$ Gabriele Crimi ${ }^{3}$ Nuccia Morici ${ }^{4}$ Tullio Palmerini ${ }^{5}$ Marco Valgimigli 6,7 \\ Stefano Savonitto ${ }^{8}$ Stefano De Servi ${ }^{2}$
}

${ }^{1}$ Division of Cardiology, Fondazione IRCCS Policlinico San Matteo, Pavia, Italy

2 Department of Molecular Cardiology, University of Pavia, Pavia, Italy

${ }^{3}$ Cardio-Thoraco-Vascular Department, Interventional Cardiology Unit, Ospedale Policlinico San Martino IRCCS, Genova, Italy

${ }^{4}$ First Division of Cardiology, Niguarda Ca'Grande Hospital, Milano, Italy

${ }^{5}$ Division of Cardiology, Policlinico Sant'Orsola, Bologna, Italy

${ }^{6}$ Department of Cardiology, CardioCentro Ticino, Lugano, Switzerland

${ }^{7}$ Department of Cardiology, University of Bern, Bern, Switzerland

8 Divison of Cardiology, Ospedale Manzoni, Lecco, Italy

Thromb Haemost 2021;121:1255-1257.

\begin{abstract}
Address for correspondence Claudio Montalto, MD, Division of Cardiology, Fondazione IRCCS Policlinico San Matteo, Viale Camillo Golgi 19, Pavia, Italy (e-mail: cm.claudio.montalto@gmail.com).
\end{abstract}

The Academic Research Consortium (ARC) proposed a definition for high bleeding risk (HBR) status to be implemented in trials. ${ }^{1}$ Recent papers validated these criteria in all-comers undergoing percutaneous coronary intervention $(\mathrm{PCI})^{2,3}$ and showed a significant increase in bleeding risk according to the number of criteria fulfilled. ${ }^{4}$ However, older adults undergoing PCI were underrepresented in validation studies of the ARC HBR score, and its external validity remains unclear in this population. $^{5}$

We analyzed a cohort including patients older $>74$ years from three large, multicenter prospective studies enrolling subjects with a final diagnosis of acute coronary syndrome (ACS) and undergoing PCI: the randomized clinical trials ElderlyACS (NCT00510185), ${ }^{6}$ Elderly-ACS 2 (NCT01777503), ${ }^{7}$ and the prospective GEPRESS study. ${ }^{8}$ Bleeding events were adjudicated according to the Bleeding Academic Research Consortium (BARC) scale. Follow-up was censored at 1 year.

In this analysis we applied the following ARC-HBR criteria: oral anticoagulant therapy; estimated glomerular filtration rate (eGFR) $<30 \mathrm{~mL} / \mathrm{min}$; baseline hemoglobin value $<11 \mathrm{~g} / \mathrm{dL}$; baseline platelet value $<100 \times 10^{9} / \mathrm{L} ;$ age $\geq 75$ years; eGFR $<60 \mathrm{~mL} / \mathrm{min}$; hemoglobin 11 to $12.9 \mathrm{~g} / \mathrm{dL}$ for men and 11 to $11.9 \mathrm{~g} / \mathrm{dL}$ for women, and previous stroke. We computed the ARC-HBR status at a patient-level according to definition described in the consensus paper, ${ }^{1}$ meaning at least one major and/or two minor criteria; we also stratified the number of

received

October 15, 2020

accepted after revision

December 19, 2020

published online

December 22, 2020 times each patient met this definition. Individual criteria were tested for association with BARC 3 or 5 events in a univariate generalized linear model if at least one event occurred in the subgroup and in a Cox multivariable model if they were univariate predictors. For comparison, also the PRECISE-DAPT ${ }^{9}$ and PARIS ${ }^{10}$ risk scores were calculated at a patient-level, imputed in a time-to-event receiver operator curve analysis and compared with ARC-HBR status. Statistical analysis was performed in the $R$ environment.

Baseline characteristics of the 1,988 subjects included are in - Supplementary Table $\mathbf{1} 1$ (available in the online version). Of them, 1,184 (59.5\%) met the ARC-HBR definition. The most common major criteria were severe-end-stage chronic kidney disease (CKD; $n=121,6.1 \%$ ) and moderate-severe anemia ( $n=110,5.5 \%$ ). Considering that age $>75$ years was present by definition, the second most common minor criterion was moderate CKD $(n=850,42.8 \%)$ followed by mild anemia ( $n=418,21.0 \%$; - Supplementary Fig. S1, available in the online version). When major and minor ARC-HBR criteria were explored individually, only moderate-severe anemia and severe-end-stage CKD were both univariate and multivariable predictors of bleeding with a comparable hazard ratio (HR; both multivariable HR 2.8 and $p=0.04$ ). Notably, only $19(1.0 \%)$ subjects presented both these criteria conjunctly. At 1 year, a total of 31 BARC 3 or 5 bleeding events occurred, 25 in the ARCHBR group and 6 in those not meeting fulfilling the criteria.
(C) 2020. Thieme. All rights reserved. DOI https://doi.org/ Georg Thieme Verlag KG,

Rüdigerstraße 14,

70469 Stuttgart, Germany 10.1055/a-1342-3750. ISSN 0340-6245. 


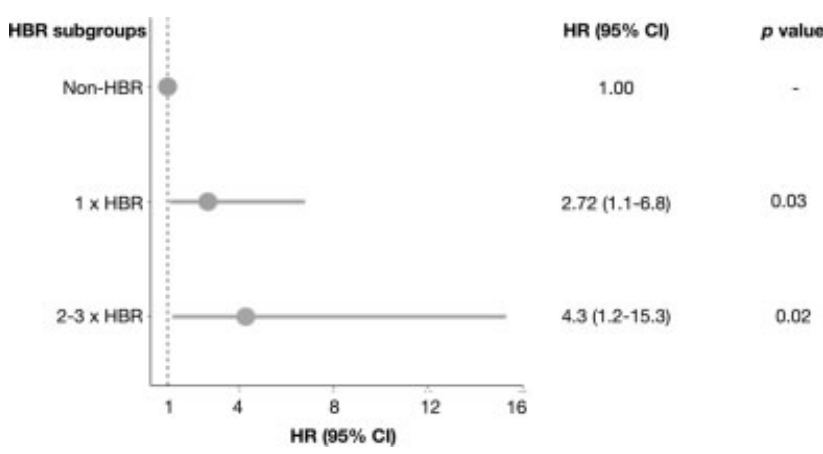

Fig. 1 Hazard ratios and 95\% confidence interval for Bleeding Academic Research Consortium (BARC) 3 or 5 bleedings according to the number of times the ARC-HBR definition was fulfilled; non-HBR is the reference. ARC-HBR, Academic Research Consortium high bleeding risk.

Patients who met the ARC-HBR definition had a significantly higher risk of BARC 3 or 5 bleedings (HR: 2.8; 95\% confidence interval 1.2-7.0; log-rank $p=0.01$; - Fig. 1). Of them, 1,053 (53.0\%) fulfilled the ARC-HBR definition once, 127 (6.4\%) twice, and $4(0.2 \%)$ three times. Compared with those with non-HBR, we observed a significantly higher hazard of bleedings with a stepwise increase in HR according to the number of times the ARC-HBR definition was fulfilled (log-rank $p=0.03$ and - Supplementary Fig. S2, available in the online version).

Area under the curve (AUC) analysis revealed a moderate discriminative power $(c$-statistic $=0.61)$ for the ARC-HBR status. When compared with other bleeding risk scores calculated, only the PRECISE-DAPT showed a significantly higher AUC ( $c$-statistic $=0.69 ; p=0.04)$ while the PARIS score showed a similar predictive power $(c$-statistic $=0.60$; $p=0.9$ ) (-Fig. 2).

In conclusion, our study observed that the ARC-HBR definition is able to identify older adults undergoing PCI who are at higher risk of bleeding. Our findings are consistent with those of another validation study that compared ARCHBR, PARIS, and PRECISE-DAPT risk scores in a larger sample of unselected patients undergoing $\mathrm{PCI},{ }^{3}$ which suggested that the ARC-HBR have a similar performance than the PARIS and PRECISE-DAPT score and therefore it could be implemented in clinical practice. These results corroborate the utility of the ARC-HBR and its widespread use in future clinical trials dedicated to elderly subjects. Of note, milder form of anemia and CKD were not independent predictors of bleeding.

We also observed that fulfilling the ARC-HBR definition (one major or two minor criteria) more than once significantly increased the hazard of bleeding, but the ARC-HBR definition retained only a modest discriminative power, which was similar for the PARIS score AUC ( $c$-statistic $=0.60$ and 0.61 , respectively). On the other hand, the PRECISE-DAPT showed a slightly higher discriminative value (c-statistic $=0.69$ ) which was significantly higher than that of ARCHBR $(p=0.04)$. Our work also suggests that the PRECISEDAPT score might offer a modestly, but significantly, improved discriminative capacity in older adults which confirms previous findings. ${ }^{11}$ The ARC-HBR definition and the PARIS score rely on broader definitions of items which

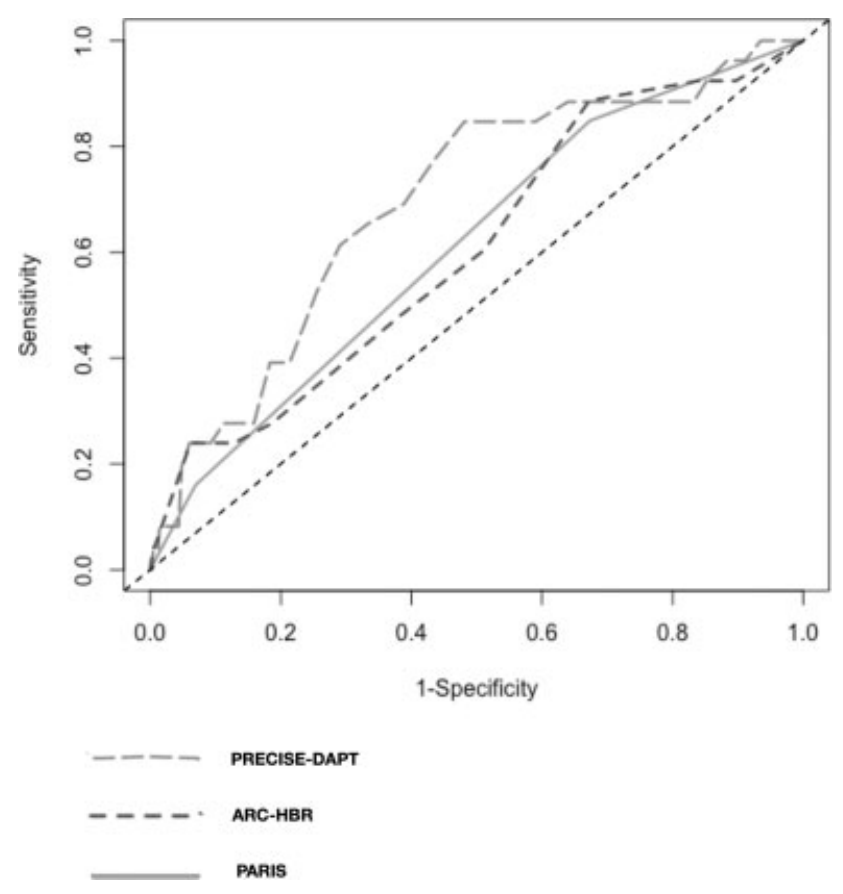

Fig. 2 ROC curve analysis for ARC-HBR, PARIS, and PRECISE-DAPT risk scores. ARC-HBR, Academic Research Consortium high bleeding risk; ROC, receiver operator curve.

might overestimate bleeding risk in older adults who often present with several risk factors in conjunction.

\section{Limitations}

Some limitations should be acknowledged. First, a relatively small number of events were observed. Second, 8 of the 20 domains proposed by the ARC-HBR definition were explored. In fact, this validation cohort of older adults includes patients from studies dated before the ARC-HBR statement, ${ }^{1}$ therefore some criteria were not prospectively collected or represented exclusion criteria for enrollment. This is a limitation found also in other published validation initiatives ${ }^{3,4}$ that might limit the validity of our findings. Third, our validation cohort enrolled patients from Western countries and this might limit the generalizability of our findings to other ethnicities; whether a population difference exists deserve further attention in future research.

\section{Funding}

None.

Conflict of Interest

None declared.

\section{References}

1 Urban P, Mehran R, Colleran R, et al. Defining high bleeding risk in patients undergoing percutaneous coronary intervention. Circulation 2019;140(03):240-261

2 Corpataux N, Spirito A, Gragnano F, et al. Validation of high bleeding risk criteria and definition as proposed by the Academic Research Consortium for high bleeding risk. Eur Heart J 2020;41 (38):3743-3749 
3 Ueki Y, Bär S, Losdat S, et al. Validation of bleeding risk criteria (ARC-HBR) in patients undergoing percutaneous coronary intervention and comparison with contemporary bleeding risk scores. EuroIntervention 2020;16(05):371-379

4 Cao D, Mehran R, Dangas G, et al. Validation of the Academic Research Consortium high bleeding risk definition in contemporary $\mathrm{PCI}$ patients. J Am Coll Cardiol 2020;75(21):2711-2722

5 Montalto C, Crimi G, Morici N, Savonitto S, De Servi S. Use of clinical risk score in an elderly population: need for ad hoc validation and calibration. J Am Coll Cardiol 2019;74(01):161-162

6 Savonitto S, Cavallini C, Petronio AS, et al; Italian Elderly ACS Trial Investigators. Early aggressive versus initially conservative treatment in elderly patients with non-ST-segment elevation acute coronary syndrome: a randomized controlled trial. JACC Cardiovasc Interv 2012;5(09):906-916

7 Savonitto S, Ferri LA, Piatti L, et al;Elderly ACS 2 Investigators. Comparison of reduced-dose prasugrel and standard-dose clopidogrel in elderly patients with acute coronary syndromes undergoing early percutaneous revascularization. Circulation 2018;137 (23):2435-2445
8 Palmerini T, Calabrò P, Piscione F, et al. Impact of gene polymorphisms, platelet reactivity, and the SYNTAX score on 1-year clinical outcomes in patients with non-ST-segment elevation acute coronary syndrome undergoing percutaneous coronary intervention: the GEPRESS study. JACC Cardiovasc Interv 2014; 7(10):1117-1127

9 Costa F, van Klaveren D, James S, et al;PRECISE-DAPT Study Investigators. Derivation and validation of the predicting bleeding complications in patients undergoing stent implantation and subsequent dual antiplatelet therapy (PRECISE-DAPT) score: a pooled analysis of individual-patient datasets from clinical trials. Lancet 2017;389(10073):1025-1034

10 Baber U, Mehran R, Giustino G, et al. Coronary thrombosis and major bleeding after PCI with drug-eluting stents: risk scores from PARIS. J Am Coll Cardiol 2016;67(19):2224-2234

11 Montalto C, Crimi G, Morici N, et al. Bleeding risk prediction in elderly patients managed invasively for acute coronary syndromes: external validation of the PRECISEDAPT and PARIS scores. Int J Cardiol 2020:S0167-5273(20) 34234-0 